\title{
A multivariate dependence measure for aggregating risks
}

\author{
Jan Dhaene $^{1} \quad$ Daniël Linders $^{2} \quad$ Wim Schoutens $^{3} \quad$ David Vyncke $^{4}$
}

December 1, 2013

${ }^{1}$ KU Leuven, Leuven, Belgium. Email: jan.dhaene@econ.kuleuven.be ${ }^{2}$ KU Leuven, Leuven, Belgium. Email: daniel.linders@econ.kuleuven.be ${ }^{3}$ KU Leuven, Leuven, Belgium. Email: wim.schoutens@wis.kuleuven.be ${ }^{4}$ Ghent University, Gent, Belgium. Email: david.vyncke@ugent.be 


\begin{abstract}
To evaluate the aggregate risk in a financial or insurance portfolio, a risk analyst has to calculate the distribution function of a sum of random variables. As the individual risk factors are often positively dependent, the classical convolution technique will not be sufficient. On the other hand, assuming a comonotonic dependence structure will likely overrate the real aggregate risk. In order to choose between both approximations, or perhaps use a weighted average, we should have an indication on the accuracy. Clearly this accuracy will depend on the copula between the individual risk factors, but it is also influenced by the marginal distributions. In this paper we introduce a multivariate dependence measure that takes both aspects into account. This new measure differs from other multivariate dependence measures, as it focuses on the aggregate risk rather than on the copula or the joint distribution function itself. We prove several interesting properties of this new measure and discuss its relation to other dependence measures. We also give some comments on the estimation and conclude with examples and numerical results.
\end{abstract}

Keywords: comonotonic copula, independence, aggregate distribution, concordance order, positive quadrant dependence 


\section{Introduction}

When evaluating the risk exposure of a financial or insurance portfolio, the risk analyst has to evaluate a sum of random variables. Consider a portfolio $\boldsymbol{X}$ consisting of $d$ risk factors $X_{1}, X_{2}, \ldots, X_{d}$, then the aggregate risk is $S=X_{1}+X_{2}+\cdots+X_{d}$. To determine the distribution of this aggregate risk, we have to know the joint distribution $F_{\boldsymbol{X}}$ of $\left(X_{1}, X_{2}, \ldots, X_{d}\right)$. In practice however this often turns out to be very difficult. Modeling the marginal distributions of $X_{i}$ is quite a common task, but finding the appropriate copula between the $X_{i}$ is much less straightforward. Moreover the calculation of the aggregate distribution involves a $d$-dimensional integral, which is not very appealing for highdimensional portfolios.

One way to tackle this problem is to simply neglect the dependence and assume that the risks are independent. Let $\boldsymbol{X}^{\perp}=\left(X_{1}^{\perp}, X_{2}^{\perp}, \ldots, X_{d}^{\perp}\right)$ be a random vector with the same marginal distributions as $\boldsymbol{X}$ but with independent components, i.e. $\boldsymbol{X}^{\perp}$ has cumulative distribution

$$
F_{\boldsymbol{X}^{\perp}}(\boldsymbol{x})=F_{X_{1}}\left(x_{1}\right) F_{X_{2}}\left(x_{2}\right) \ldots F_{X_{d}}\left(x_{d}\right) .
$$

The distribution of $S^{\perp}=X_{1}^{\perp}+X_{2}^{\perp}+\cdots+X_{d}^{\perp}$ can be obtained by the well-known convolution technique or, for some specific marginal distributions, by the recursion formulas in Panjer (1981) and others. Obviously, neglecting the (usually positive) dependence, we might underrate the aggregate risk as $S^{\perp}$ will usually have a smaller variance. Note however that $\mathrm{E}[S]=\mathrm{E}\left[S^{\perp}\right]$, as $\boldsymbol{X}$ and $\boldsymbol{X}^{\perp}$ belong to the same Fréchet class.

Alternatively, one might consider the strongest positive dependence and assume that the risks are comonotonic. Let $\boldsymbol{X}^{c}=\left(X_{1}^{c}, X_{2}^{c}, \ldots, X_{d}^{c}\right)$ be a random vector with the same marginal distributions as $\boldsymbol{X}$ but with comonotonic components, i.e. $\boldsymbol{X}^{c}$ has cumulative distribution

$$
F_{X^{c}}(\boldsymbol{x})=\min \left\{F_{X_{1}}\left(x_{1}\right), F_{X_{2}}\left(x_{2}\right), \ldots, F_{X_{d}}\left(x_{d}\right)\right\}
$$

or, equivalently,

$$
\boldsymbol{X}^{c}={ }_{d}\left(F_{X_{1}}^{-1}(U), F_{X_{2}}^{-1}(U), \ldots, F_{X_{d}}^{-1}(U)\right), \quad U \sim U(0,1)
$$

where $={ }_{d}$ denotes equality in distribution. The distribution function of $S^{c}=X_{1}^{c}+X_{2}^{c}+\cdots+X_{d}^{c}$ can be obtained by inverting the quantile function, which in turn equals the sum of the marginal quantile functions $F_{X_{i}}^{-1}$. Dhaene et al. (2002a) show that $S$ is smaller in convex order than $S^{c}$ (written $S \leq_{c x} S^{c}$ ), i.e.

$$
\mathrm{E}[v(S)] \leq \mathrm{E}\left[v\left(S^{c}\right)\right]
$$

for all real convex functions $v$, provided the expectations exist. This implies that $S^{c}$ has heavier tails than $S$ and $\operatorname{Var}\left[S^{c}\right] \geq \operatorname{Var}[S]$, so the aggregate risk will likely be overrated. Note that $\boldsymbol{X}$ and $\boldsymbol{X}^{c}$ also belong to the same Fréchet class, so $\mathrm{E}[S]=\mathrm{E}\left[S^{c}\right]$.

In order to choose between both approximations, or perhaps use a weighted average, we should have an indication on the accuracy. Clearly this accuracy will depend on the copula of $\boldsymbol{X}$, but it is also influenced by the marginal distributions. In this paper we introduce a multivariate dependence measure that takes both aspects into account. This new measure differs from other multivariate dependence measures in e.g. Wolff (1980), Fernández Fernández and González-Barrios (2004), Taylor (2007), Behboodian et al. (2007), Schmid and Schmidt (2007) or Koch and De Schepper (2011), as it focuses on the aggregate risk $S$ rather than on the copula or the joint distribution function of $\boldsymbol{X}$. In a finance context, it can be translated into a measure for herd behavior, see Dhaene et al. (2012).

In the following section we introduce the new multivariate dependence measure and prove several interesting properties. We also discuss its relation to the classical Pearson correlation and the comonotonicity coefficient of Koch and De Schepper (2011). In section 3 we give some comments on the estimation and section 4 concludes with examples and numerical results. 


\section{Definition and properties}

Most of the multivariate dependence measures proposed in literature are written directly in terms of the copula or the joint distribution function of $\boldsymbol{X}$. Keeping the aggregate risk in mind, we propose to measure the dependence in $\boldsymbol{X}$ indirectly through the distribution of the sum $S$ of its components. More specifically, we will focus on the variance of $S$. As convex order implies ordered variances, we have that $\operatorname{Var}(S) \leq \operatorname{Var}\left(S^{c}\right)$. This suggests the following multivariate dependence measure.

Definition 2.1 The dependence measure $\rho_{c}$ of a random vector $\boldsymbol{X}$ with non-degenerate margins is defined as

$$
\rho_{c}(\boldsymbol{X})=\frac{\operatorname{Var}(S)-\operatorname{Var}\left(S^{\perp}\right)}{\operatorname{Var}\left(S^{c}\right)-\operatorname{Var}\left(S^{\perp}\right)}=\frac{\sum_{i=1}^{d} \sum_{j<i} \operatorname{Cov}\left(X_{i}, X_{j}\right)}{\sum_{i=1}^{d} \sum_{j<i} \operatorname{Cov}\left(X_{i}^{c}, X_{j}^{c}\right)}
$$

provided the covariances exist.

The first expression in (1) has a similar structure as the multivariate dependence measures $\rho_{n}$ in Wolff (1980) and $\kappa$ in Koch and De Schepper (2011). Both measures are also centered around the independent vector and normalized with respect to the comonotonic vector. From the second expression we see that $\rho_{c}$ can be interpreted as a normalized average of bivariate covariances. Since the numerator cannot exceed the denominator, $\rho_{c}$ is bounded from above by 1 , see Proposition 2.8. Without imposing some restrictions on $\boldsymbol{X}$ however, there is no general lower bound, see Proposition 2.9.

The condition of non-degenerate margins ensures that the denominator in (1) is non-zero. Before we prove this assertion, we extend a result of Luan (2001) for positive random variables to real-valued random variables. The proof in Luan (2001) relies on the assumption that $X$ and $Y$ are bounded from below, so we give a somewhat different proof.

Lemma 2.2 Two random variables $X$ and $Y$ are both independent and comonotonic if and only if at least one of them is degenerate.

Proof. First, assume that $Y$ is degenerate with value $a$, i.e. $\mathrm{P}(Y=a)=1$ and $\mathrm{P}(Y \neq a)=0$. Then,

$$
F_{X, Y}(x, y)=\mathrm{P}(X \leq x, Y \leq y)= \begin{cases}0 & y<a \\ F_{X}(x) & y \geq a\end{cases}
$$

and

$$
\min \left(F_{X}(x), F_{Y}(y)\right)=F_{X}(x) F_{Y}(y)= \begin{cases}0 & y<a \\ F_{X}(x) & y \geq a\end{cases}
$$

so $X$ and $Y$ are both independent and comonotonic.

Conversely, assume that $X$ and $Y$ are both independent and comonotonic. Without loss of generality, assume that $X$ is non-degenerate. Hence, there is a least one value $x$ for which $0<F_{X}(x)<1$. Since $X$ and $Y$ are independent and comonotonic, we have

$$
F_{X, Y}(x, y)=\min \left(F_{X}(x), F_{Y}(y)\right)=F_{X}(x) F_{Y}(y), \quad \forall x, y
$$

For fixed $x$ with $0<F_{X}(x)<1, F_{Y}(y)<F_{X}(x)$ then implies $F_{Y}(y)=F_{X}(x) F_{Y}(y)$ and thus $F_{Y}(y)=0$ because $F_{X}(x) \neq 1$. On the other hand, $F_{Y}(y)>F_{X}(x)$ implies $F_{X}(x)=F_{X}(x) F_{Y}(y)$ and thus $F_{Y}(y)=1$, because $F_{X}(x) \neq 0$. The third case $F_{Y}(y)=F_{X}(x)$ would imply $F_{X}(x)=\left(F_{X}(x)\right)^{2}$ which contradicts $0<F_{X}(x)<1$. Consequently, $F_{Y}(y)$ is either 0 or 1 and thus $Y$ is a degenerate random variable.

We also need the notion of positive quadrant dependence, see e.g. Lehmann (1966). 
Definition 2.3 A random couple $(X, Y)$ is said to be positively quadrant dependent (PQD) if

$$
\mathrm{P}(X \leq x, Y \leq y) \geq \mathrm{P}(X \leq x) \mathrm{P}(Y \leq y), \quad \text { for all }(x, y) \in \mathbb{R}^{2},
$$

This bivariate notion of dependence can be generalized to higher dimensions by defining positive orthant dependence, see e.g. Denuit et al. (2006).

Definition 2.4 A random vector $\left(X_{1}, X_{2} \ldots, X_{d}\right)$ is said to be positively lower orthant dependent (PLOD) if

$$
\mathrm{P}\left(X_{1} \leq x_{1}, \ldots, X_{d} \leq x_{d}\right) \geq \prod_{i=1}^{d} \mathrm{P}\left(X_{i} \leq x_{i}\right), \quad \text { for all }\left(x_{1}, x_{2}, \ldots, x_{d}\right) \in \mathbb{R}^{d}
$$

It is said to be positively upper orthant dependent (PUOD) if

$$
\mathrm{P}\left(X_{1}>x_{1}, \ldots, X_{d}>x_{d}\right) \geq \prod_{i=1}^{d} \mathrm{P}\left(X_{i}>x_{i}\right), \quad \text { for all }\left(x_{1}, x_{2}, \ldots, x_{d}\right) \in \mathbb{R}^{d}
$$

When both (2) and (3) hold, the random vector is called positively orthant dependent (POD).

Theorem 2.5 For any random vector $\boldsymbol{X}=\left(X_{1}, X_{2} \ldots, X_{d}\right)$, the following expressions are equivalent:

(i) $X_{1}^{c}+X_{2}^{c}+\ldots+X_{d}^{c}={ }_{d} X_{1}^{\perp}+X_{2}^{\perp}+\ldots+X_{d}^{\perp}$

(ii) $\operatorname{Var}\left(X_{1}^{c}+X_{2}^{c}+\ldots+X_{d}^{c}\right)=\operatorname{Var}\left(X_{1}^{\perp}+X_{2}^{\perp}+\ldots+X_{d}^{\perp}\right)$

(iii) At least $d-1$ marginal variables $X_{j}$ are degenerate.

Proof. The proofs of (i) $\Rightarrow$ (ii) and (iii) $\Rightarrow$ (ii) are straightforward.

(ii) $\Rightarrow$ (i): Dhaene et al. (2002a) show that $X=_{d} Y$ if and only if $X \leq_{c x} Y$ and $\operatorname{Var}(X)=\operatorname{Var}(Y)$ so it suffices to note that $X_{1}^{\perp}+X_{2}^{\perp}+\ldots+X_{d}^{\perp} \leq_{c x} X_{1}^{c}+X_{2}^{c}+\ldots+X_{d}^{c}$.

(ii) $\Rightarrow$ (iii): we know from Hoeffding (1940), see also Lehmann (1966), that for any two random variables $X$ and $Y$ with joint distribution function $F_{X, Y}$ and marginal distribution functions $F_{X}$ and $F_{Y}$,

$$
\operatorname{Cov}(X, Y)=\int_{-\infty}^{\infty} \int_{-\infty}^{\infty}\left(F_{X, Y}(x, y)-F_{X}(x) F_{X}(y)\right) d x d y
$$

This implies that $\operatorname{Cov}\left(X_{i}^{\perp}, X_{j}^{\perp}\right) \leq \operatorname{Cov}\left(X_{i}^{c}, X_{j}^{c}\right), \forall i, j$. Since

$$
\sum_{i=1}^{d} \sum_{j=1}^{d} \operatorname{Cov}\left(X_{i}^{\perp}, X_{j}^{\perp}\right)=\sum_{i=1}^{d} \sum_{j=1}^{d} \operatorname{Cov}\left(X_{i}^{c}, X_{j}^{c}\right),
$$

we have that $\operatorname{Cov}\left(X_{i}^{c}, X_{j}^{c}\right)=\operatorname{Cov}\left(X_{i}^{\perp}, X_{j}^{\perp}\right)$ for all $i$ and $j$, and thus $\operatorname{Cov}\left(X_{i}^{c}, X_{j}^{c}\right)=0$ for all $i \neq j$. From lemma 3 in Lehmann (1966) we know that random variables that are PQD and uncorrelated are independent. Clearly, the couple $\left(X_{i}^{c}, X_{j}^{c}\right)$ is $\mathrm{PQD}$, so $\operatorname{Cov}\left(X_{i}^{c}, X_{j}^{c}\right)=0$ implies that $X_{i}^{c}$ and $X_{j}^{c}(i \neq j)$ are both comonotonic and independent. Lemma 2.2 then ensures that if $X_{i}$ is non-degenerate for fixed $i$, all $X_{j}$ with $j \neq i$ are degenerate.

Our new dependence measure has several interesting properties. For instance, it satisfies the axioms of normalization, monotonicity, permutation invariance and duality in Taylor (2007). Before we discuss these properties, we recall the notion of concordance order, see e.g. Müller and Scarsini (2000). 
Definition 2.6 A random vector $\boldsymbol{X}=\left(X_{1}, X_{2}, \ldots, X_{d}\right)$ is said to be smaller than the random vector $\boldsymbol{Y}=$ $\left(Y_{1}, Y_{2}, \ldots, Y_{d}\right)$ in the concordance order, written $\boldsymbol{X} \leq_{c} \boldsymbol{Y}$, if both

$$
\mathrm{P}\left(X_{1} \leq t_{1}, X_{2} \leq t_{2}, \ldots, X_{d} \leq t_{d}\right) \leq \mathrm{P}\left(Y_{1} \leq t_{1}, Y_{2} \leq t_{2}, \ldots, Y_{d} \leq t_{d}\right)
$$

and

$$
\mathrm{P}\left(X_{1}>t_{1}, X_{2}>t_{2}, \ldots, X_{d}>t_{d}\right) \leq \mathrm{P}\left(Y_{1}>t_{1}, Y_{2}>t_{2}, \ldots, Y_{d}>t_{d}\right)
$$

hold for all $\left(t_{1}, t_{2}, \ldots, t_{d}\right) \in \mathbb{R}^{d}$.

Theorem 2.7 For any two random vectors $\boldsymbol{X}=\left(X_{1}, X_{2}, \ldots, X_{d}\right)$ and $\boldsymbol{Y}=\left(Y_{1}, Y_{2}, \ldots, Y_{d}\right), \rho_{c}$ has the following properties:

(i) (normalization) If $\boldsymbol{X}$ has comonotonic components, then $\rho_{c}(\boldsymbol{X})=1$; if $\boldsymbol{X}$ has independent components, then $\rho_{c}(\boldsymbol{X})=0$.

(ii) (monotonicity) If $\boldsymbol{X}$ is smaller than $\boldsymbol{Y}$ in the concordance order, then $\rho_{c}(\boldsymbol{X}) \leq \rho_{c}(\boldsymbol{Y})$.

(iii) (permutation invariance) For any permutation $\left(i_{1}, i_{2}, \ldots, i_{d}\right)$ of $(1,2, \ldots, d)$, we have that $\rho_{c}\left(X_{i_{1}}, X_{i_{2}}, \ldots, X_{i_{d}}\right)=$ $\rho_{c}\left(X_{1}, X_{2}, \ldots, X_{d}\right)$.

(iv) (duality) $\rho_{c}\left(-X_{1},-X_{2}, \ldots,-X_{d}\right)=\rho_{c}\left(X_{1}, X_{2}, \ldots, X_{d}\right)$.

Proof. The proofs of (i) and (iii) are straightforward.

(ii) Random vectors that are ordered in concordance order obviously have the same marginal distributions. Consequently, $\left(X_{i}^{c}, X_{j}^{c}\right)$ and $\left(Y_{i}^{c}, Y_{j}^{c}\right)$ have the same distribution and

$$
\sum_{i=1}^{d} \sum_{j<i} \operatorname{Cov}\left(X_{i}^{c}, X_{j}^{c}\right)=\sum_{i=1}^{d} \sum_{j<i} \operatorname{Cov}\left(Y_{i}^{c}, Y_{j}^{c}\right)
$$

so $\rho_{c}(\boldsymbol{X})$ and $\rho_{c}(\boldsymbol{Y})$ have the same denominator. On the other hand, $\boldsymbol{X} \leq_{c} \boldsymbol{Y}$ implies that

$$
F_{X_{i}, X_{j}}\left(t_{i}, t_{j}\right) \leq F_{Y_{i}, Y_{j}}\left(t_{i}, t_{j}\right), \quad 1 \leq i, j \leq d, t_{i}, t_{j} \in \mathbb{R} .
$$

Using (4) we then find that $\operatorname{Cov}\left(X_{i}, X_{j}\right) \leq \operatorname{Cov}\left(Y_{i}, Y_{j}\right)$ for all $i$ and $j$. Hence,

$$
\sum_{i=1}^{d} \sum_{j<i} \operatorname{Cov}\left(X_{i}, X_{j}\right) \leq \sum_{i=1}^{d} \sum_{j<i} \operatorname{Cov}\left(Y_{i}, Y_{j}\right)
$$

which concludes the proof.

(iv) Clearly, $\operatorname{Cov}\left(-X_{i},-X_{j}\right)=\operatorname{Cov}\left(X_{i}, X_{j}\right)$ for all $i$ and $j$. For the comonotonic vector we find

$$
\begin{aligned}
\operatorname{Cov}\left(\left(-X_{i}\right)^{c},\left(-X_{j}\right)^{c}\right) & =\operatorname{Cov}\left(F_{-X_{i}}^{-1}(U), F_{-X_{j}}^{-1}(U)\right), \quad U \sim U(0,1) \\
& =\operatorname{Cov}\left(-F_{X_{i}}^{-1}(1-U),-F_{X_{j}}^{-1}(1-U)\right) \\
& =\operatorname{Cov}\left(F_{X_{i}}^{-1}(V), F_{X_{j}}^{-1}(V)\right), \quad V \sim U(0,1) \\
& =\operatorname{Cov}\left(X_{i}^{c}, X_{j}^{c}\right)
\end{aligned}
$$

Hence,

$$
\rho_{c}\left(-X_{1},-X_{2}, \ldots,-X_{d}\right)=\frac{\sum_{i=1}^{d} \sum_{j<i} \operatorname{Cov}\left(-X_{i},-X_{j}\right)}{\sum_{i=1}^{d} \sum_{j<i} \operatorname{Cov}\left(\left(-X_{i}\right)^{c},\left(-X_{j}\right)^{c}\right)}=\frac{\sum_{i=1}^{d} \sum_{j<i} \operatorname{Cov}\left(X_{i}, X_{j}\right)}{\sum_{i=1}^{d} \sum_{j<i} \operatorname{Cov}\left(X_{i}^{c}, X_{j}^{c}\right)}
$$

which equals $\rho_{c}\left(X_{1}, X_{2}, \ldots, X_{d}\right)$ by definition. 
Theorem 2.7(i) states that $\rho_{c}$ equals 1 if the components of $\boldsymbol{X}$ are maximally dependent. We can show that the reverse implication also holds. Moreover, the value $\rho_{c}=1$ is maximal.

Proposition 2.8 For any random vector $\boldsymbol{X}$ we have $\rho_{c}(\boldsymbol{X}) \leq 1$. If $\rho_{c}(\boldsymbol{X})=1$, then $\boldsymbol{X}={ }_{d} \boldsymbol{X}^{c}$.

Proof. From (4) and the fact that $F_{X, Y}(x, y) \leq \min \left(F_{X}(x), F_{Y}(y)\right), \forall x, y$, it follows that

$$
\operatorname{Cov}(X, Y) \leq \operatorname{Cov}\left(X^{c}, Y^{c}\right)
$$

for any random couple $(X, Y)$, with equality if and only if $X$ and $Y$ are comonotonic. Consequently, $\operatorname{Cov}\left(X_{i}, X_{j}\right) \leq \operatorname{Cov}\left(X_{i}^{c}, X_{j}^{c}\right)$ for any $\left(X_{i}, X_{j}\right)$ and equation (1) then implies that $\rho_{c}(\boldsymbol{X}) \leq 1$.

If $\rho_{c}(\boldsymbol{X})=1$, then all $\operatorname{Cov}\left(X_{i}, X_{j}\right)$ must be equal to $\operatorname{Cov}\left(X_{i}^{c}, X_{j}^{c}\right)$ and thus $\left(X_{i}, X_{j}\right)$ are comonotonic for all $i$ and $j$. From Theorem 4 in Dhaene et al. (2002a) we know that comonotonicity of a random vector is equivalent with pairwise comonotonicity of its components, so $\boldsymbol{X}={ }_{d} \boldsymbol{X}^{c}$.

The reverse implication for $\rho_{c}=0$ does not hold in general. Indeed, one can easily construct a nonindependent random vector for which $\rho_{c}=0$. Consider e.g. a multivariate normal vector $\left(X_{1}, X_{2}, X_{3}\right)$ with covariance matrix

$$
\left(\begin{array}{ccc}
1 & -0.4 & -0.4 \\
-0.4 & 1 & 0.8 \\
-0.4 & 0.8 & 1
\end{array}\right)
$$

The normally distributed variables $X_{1}, X_{2}$ and $X_{3}$ are clearly not independent and yet $\rho_{c}=0$ because of the presence of both positive and negative dependence. In the following proposition, we exclude the possibility of negative dependence by considering only random vectors that are pairwise PQD. In this rather weak dependence structure all couples $\left(X_{i}, X_{j}\right), 1 \leq i, j \leq d$, are positively quadrant dependent.

Proposition 2.9 For any pairwise PQD random vector $\boldsymbol{X}$ we have $\rho_{c}(\boldsymbol{X}) \geq 0$. If $\rho_{c}(\boldsymbol{X})=0$, then all $X_{i}$ and $X_{j}(i \neq j)$ are pairwise independent.

Proof. As each couple $\left(X_{i}, X_{j}\right)$ is $\mathrm{PQD}$ it follows from (4) that $\operatorname{Cov}\left(X_{i}, X_{j}\right) \geq 0,1 \leq i, j \leq d$. Consequently, $\rho_{c}(\boldsymbol{X}) \geq 0$ and if $\rho_{c}(\boldsymbol{X})=0$, then $\operatorname{Cov}\left(X_{i}, X_{j}\right)=0$ for all $i \neq j$. Lemma 3 in Lehmann (1966) then ensures that all $X_{i}$ and $X_{j}(i \neq j)$ are independent.

Note that for mutual independence one needs a stronger positive dependence notion. E.g. Joag-Dev (1983) shows that pairwise independence implies mutual independence if $\boldsymbol{X}$ is associated or strongly positively orthant dependent.

Next we study the relation of $\rho_{c}$ to the classical Pearson correlation $r$. Although widely used, this dependence measure is often misinterpreted as it measures only linear dependence, see e.g. Embrechts et al. (2002). Moreover, Shih and Huang (1992) have noticed that, unless the marginal distributions of the two random variables can be different only in location or scale parameters, the range of $r$ is smaller than the usual reference interval $[-1,1]$. In the following proposition we show that $\rho_{c}$ for $d=2$ is equal to the Pearson correlation $r$, but only when the marginal distributions allow for linear dependence.

Proposition 2.10 For any random couple $(X, Y)$ we have that $\rho_{c}(X, Y)=r(X, Y)$ if and only if the marginal distributions differ only in location and/or scale parameters.

Proof. The couples $(X, Y)$ and $\left(X^{c}, Y^{c}\right)$ have the same marginals, so

$$
\rho_{c}(X, Y)=\frac{\operatorname{Cov}(X, Y)}{\operatorname{Cov}\left(X^{c}, Y^{c}\right)}=\frac{r(X, Y)}{r\left(X^{c}, Y^{c}\right)}
$$


which equals $r(X, Y)$ if and only if $r\left(X^{c}, Y^{c}\right)=1$. Hence, $Y^{c}=a X^{c}+b$ with probability 1 for some constants $a>0$ and $b \in \mathbb{R}$, and thus $F_{Y}^{-1}(p)=a F_{X}^{-1}(p)+b$ from which we can conclude that the marginal distributions differ only in location and/or scale parameters.

Vyncke (2004) gives several location/scale-families in this context, e.g. exponential, normal, Rayleigh, Gumbel, Pareto (with fixed first parameter), etc. When the marginal distributions both belong to one of these families, then $\rho_{c}$ equals the Pearson correlation $r$. On the other hand, two lognormal variables with different parameters $\sigma_{1}$ and $\sigma_{2}$ can not be linearly dependent, so $\rho_{c}$ will be larger than $r$.

For $d=2$ we can relate $\rho_{c}$ also to the comonotonicity coefficient of Koch and De Schepper (2011). In essence, this multivariate dependence measure is defined as the ratio of the hypervolume between $F_{\boldsymbol{X}}$ and $F_{\boldsymbol{X}^{\perp}}$, and the hypervolume between $F_{\boldsymbol{X}^{c}}$ and $F_{\boldsymbol{X}^{\perp}}$.

Proposition 2.11 For any random couple $(X, Y)$ we have $\rho_{c}(X, Y)=\kappa(X, Y)$.

Proof. From the definition of $\rho_{c}$ and equation (4) we find that

$$
\rho_{c}(X, Y)=\frac{\operatorname{Cov}(X, Y)}{\operatorname{Cov}\left(X^{c}, Y^{c}\right)}=\frac{\int_{-\infty}^{\infty} \int_{-\infty}^{\infty}\left(F_{X, Y}(x, y)-F_{X}(x) F_{Y}(y)\right) d x d y}{\int_{-\infty}^{\infty} \int_{-\infty}^{\infty}\left(\min \left(F_{X}(x), F_{Y}(y)\right)-F_{X}(x) F_{Y}(y)\right) d x d y}
$$

in which we recognize the comonotonicity coefficient $\kappa$ for $d=2$.

Koch and De Schepper (2011) show that, if the joint distribution $\boldsymbol{X}$ can be decomposed as a convex combination of the independent cdf and the comonotonic cdf, $\kappa(\boldsymbol{X})$ is given by the weight of the comonotonic part. The same result holds for $\rho_{c}(\boldsymbol{X})$.

Proposition 2.12 If the joint distribution function $F_{\boldsymbol{X}}$ of $\boldsymbol{X}$, can be written as a convex combination of $F_{\boldsymbol{X}^{c}}$ and $F_{\boldsymbol{X}^{\perp}}$, i.e.

$$
F_{\boldsymbol{X}}(\boldsymbol{x})=\alpha F_{\boldsymbol{X}^{c}}(\boldsymbol{x})+(1-\alpha) F_{\boldsymbol{X}^{\perp}}(\boldsymbol{x}), \quad 0 \leq \alpha \leq 1, \forall \boldsymbol{x}
$$

then $\rho_{c}(\boldsymbol{X})=\alpha$.

Proof. Setting all $x_{k}$ equal to $\infty$ in (5), except $x_{i}$ and $x_{j}$, shows that the same convex combination holds for all bivariate marginal distributions, and hence

$$
\begin{aligned}
F_{X_{i}, X_{j}}\left(x_{i}, x_{j}\right) & =\alpha F_{X_{i}^{c}, X_{j}^{c}}\left(x_{i}, x_{j}\right)+(1-\alpha) F_{X_{i}^{\perp}, X_{j}^{\perp}}\left(x_{i}, x_{j}\right), \quad 0 \leq \alpha \leq 1 \\
& =\alpha\left(\min \left(F_{X_{i}}\left(x_{i}\right), F_{X_{j}}\left(x_{j}\right)\right)-F_{X_{i}}\left(x_{i}\right) F_{X_{j}}\left(x_{j}\right)\right)+F_{X_{i}}\left(x_{i}\right) F_{X_{j}}\left(x_{j}\right)
\end{aligned}
$$

for all $i, j$ and $x_{i}, x_{j}$. This implies that

$$
\begin{aligned}
\rho_{c}(\boldsymbol{X})= & \frac{\sum_{i=1}^{d} \sum_{j<i} \int_{-\infty}^{\infty} \int_{-\infty}^{\infty}\left(F_{X_{i}, X_{j}}(x, y)-F_{X_{i}}(x) F_{X_{j}}(y)\right) d x d y}{\sum_{i=1}^{d} \sum_{j<i} \int_{-\infty}^{\infty} \int_{-\infty}^{\infty}\left(\min \left(F_{X_{i}}(x), F_{X_{j}}(y)\right)-F_{X_{i}}(x) F_{X_{j}}(y)\right) d x d y} \\
= & \frac{\sum_{i=1}^{d} \sum_{j<i} \int_{-\infty}^{\infty} \int_{-\infty}^{\infty} \alpha\left(\min \left(F_{X_{i}}(x), F_{X_{j}}(y)\right)-F_{X_{i}}(x) F_{X_{j}}(y)\right) d x d y}{\sum_{i=1}^{d} \sum_{j<i} \int_{-\infty}^{\infty} \int_{-\infty}^{\infty}\left(\min \left(F_{X_{i}}(x), F_{X_{j}}(y)\right)-F_{X_{i}}(x) F_{X_{j}}(y)\right) d x d y} \\
= & \alpha
\end{aligned}
$$


Next we examine the effect of adding a variable $X_{d+1}$ to the random vector $\left(X_{1}, X_{2}, \ldots, X_{d}\right)$. If $X_{d+1}$ is independent of $X_{1}, X_{2}, \ldots, X_{d}$, the total amount of dependence does not change. Hence, dependence measures measuring the absolute amount of dependence should not change either, see e.g. Fernández Fernández and González-Barrios (2004). On the other hand, when we take into account the dimension of $\boldsymbol{X}$, the relative amount of dependence obviously decreases by adding an independent variable. This behavior is reflected in $\rho_{c}$.

Proposition 2.13 For any random vector $\left(X_{1}, X_{2}, \ldots, X_{d}\right)$ and any random variable $X_{d+1}$ independent of $X_{1}, X_{2}, \ldots, X_{d}$, we have

$$
\left|\rho_{c}\left(X_{1}, X_{2}, \ldots, X_{d}\right)\right| \geq\left|\rho_{c}\left(X_{1}, X_{2}, \ldots, X_{d}, X_{d+1}\right)\right|
$$

Proof. This follows immediately from

$$
\sum_{j<d+1} \operatorname{Cov}\left(X_{d+1}, X_{j}\right)=0
$$

and

$$
\sum_{i=1}^{d+1} \sum_{j<i} \operatorname{Cov}\left(X_{i}^{c}, X_{j}^{c}\right) \geq \sum_{i=1}^{d} \sum_{j<i} \operatorname{Cov}\left(X_{i}^{c}, X_{j}^{c}\right)>0
$$

\section{Estimation}

In this section we give some comments on the estimation of $\rho_{c}$ from a sample of $\boldsymbol{X}$. This might be used to estimate the dependence in a real-life dataset, but it provides also a computationally convenient way to calculate $\rho_{c}$ when the (co)variances are hard to find. In that case one could try to generate a sample from $\boldsymbol{X}$ and estimate $\rho_{c}$ from that sample.

A straightforward way to estimate $\rho_{c}$ is to replace the variances in (1) by their sample version. Consider a $d$-dimensional sample $\left\{\left(x_{i 1}, \ldots, x_{i d}\right)\right\}_{i=1, \ldots, n}$, of size $n$ and denote

$$
\begin{aligned}
\bar{x}_{j} & =\frac{1}{n} \sum_{i=1}^{n} x_{i j}, \quad j=1, \ldots, d \\
s_{i} & =\sum_{j=1}^{d} x_{i j}, \quad i=1, \ldots, n \\
\bar{s} & =\frac{1}{n} \sum_{i=1}^{n} s_{i}=\frac{1}{n} \sum_{i=1}^{n} \sum_{j=1}^{d} x_{i j}=\sum_{j=1}^{d} \bar{x}_{j}
\end{aligned}
$$

$\operatorname{Var}(S)$ and $\operatorname{Var}\left(S^{\perp}\right)$ can then be estimated by

$$
\frac{1}{n-1} \sum_{i=1}^{n}\left(s_{i}-\bar{s}\right)^{2} \quad \text { and } \quad \sum_{j=1}^{d} \frac{1}{n-1} \sum_{i=1}^{n}\left(x_{i j}-\bar{x}_{j}\right)^{2}
$$

respectively, since $\operatorname{Var}\left(S^{\perp}\right)=\sum_{j=1}^{d} \operatorname{Var}\left(X_{j}\right)$.

For the estimation of $\operatorname{Var}\left(S^{c}\right)$ we need a sample of $S^{c}$ or, alternatively, of $\boldsymbol{X}^{c}$. Dhaene et al. (2002a) show that for any $\boldsymbol{x}$ and $\boldsymbol{y}$ in the range of a comonotonic vector either $\boldsymbol{x} \leq \boldsymbol{y}$ or $\boldsymbol{y} \leq \boldsymbol{x}$ holds. In other words, all possible outcomes of $\boldsymbol{X}^{c}$ are ordered componentwise. As $\boldsymbol{X}$ and $\boldsymbol{X}^{c}$ also have the same marginal distributions, we can easily turn the sample of $\boldsymbol{X}$ into a sample of $\boldsymbol{X}^{c}$. Denoting the $i$-th order statistic of $X_{j}$ by $x_{(i) j}$, we find the following sample of $\boldsymbol{X}^{c}:\left\{\left(x_{(i) 1}, \ldots, x_{(i) d}\right)\right\}_{i=1, \ldots, n}$. Accordingly, $\left\{x_{(i) 1}+\ldots+x_{(i) d}\right\}_{i=1, \ldots, n}$ constitutes a sample of $S^{c}$. 
This sample also follows from the additivity of the quantile function for comonotonic variables

$$
F_{S^{c}}^{-1}(p)=\sum_{j=1}^{d} F_{X_{j}}^{-1}(p), \quad p \in(0,1)
$$

Replacing the quantile function by its empirical counterpart and setting $p=\frac{i-0.5}{n}$ we find

$$
s_{(i)}=\hat{F}_{S^{c}}^{-1}\left(\frac{i-0.5}{n}\right)=\sum_{j=1}^{d} \hat{F}_{X_{j}}^{-1}\left(\frac{i-0.5}{n}\right)=\sum_{j=1}^{d} x_{(i) j}, \quad i=1, \ldots, n
$$

Since

$$
\bar{s}^{c}=\frac{1}{n} \sum_{i=1}^{n} \sum_{j=1}^{d} x_{(i) j}=\frac{1}{n} \sum_{i=1}^{n} \sum_{j=1}^{d} x_{i j}=\bar{s}
$$

$\operatorname{Var}\left(S^{c}\right)$ can thus be estimated by

$$
\frac{1}{n-1} \sum_{i=1}^{n} \sum_{j=1}^{d}\left(x_{(i) j}-\bar{s}\right)^{2}
$$

Summarizing, we find the following estimator for $\rho_{c}$ :

$$
\hat{\rho}_{c}=\frac{\sum_{i=1}^{n}\left[\left(\sum_{j=1}^{d} x_{i j}-\bar{s}\right)^{2}-\sum_{j=1}^{d}\left(x_{i j}-\bar{x}_{j}\right)^{2}\right]}{\sum_{i=1}^{n}\left[\left(\sum_{j=1}^{d} x_{(i) j}-\bar{s}\right)^{2}-\sum_{j=1}^{d}\left(x_{i j}-\bar{x}_{j}\right)^{2}\right]}
$$

\section{Examples}

In this section we calculate $\rho_{c}$ for some specific random vectors. We start with a multivariate normal distribution and compare $\rho_{c}$ with the particular case discussed in Koch and De Schepper (2011).

Example 4.1 Consider a random vector $\boldsymbol{X}$ following a multivariate normal distribution with parameters $\mathrm{E}\left(X_{i}\right)=\mu_{i}, \operatorname{Var}\left(X_{i}\right)=\sigma_{i}^{2}$ and $\rho\left(X_{i}, X_{j}\right)=\rho_{i j}$. One can easily check that

$$
\rho_{c}(\boldsymbol{X})=\frac{\sum_{i=1}^{d} \sum_{j<i} \rho_{i j} \sigma_{i} \sigma_{j}}{\sum_{i=1}^{d} \sum_{j<i} \sigma_{i} \sigma_{j}}
$$

The dependence in $\boldsymbol{X}$ is clearly increasing in $\rho_{i j}$ and so does $\rho_{c}$. We also observe that $\rho_{c}$ does not depend on $\mu_{i}$.

Koch and De Schepper (2011) discuss a random vector $\boldsymbol{X}$ with $X_{i}=\sum_{k=1}^{i} Z_{k}$ where $Z_{k}$ are i.i.d. standard normal variables. These independent increments $Z_{i}$ can be interpreted as the rates of return on some investment over consecutive time periods $\left[t_{i-1}, t_{i}\right]$. The variables $X_{i}$ then give the accumulated rates of return over the time periods $\left[0, t_{i}\right]$. Although the increments $Z_{i}$ are independent, the accumulated rates of return are clearly strongly dependent. It is straightforward to show that $\boldsymbol{X}$ has a multivariate normal distribution with $\mu_{i}=0, \sigma_{i}^{2}=i$ and $\operatorname{Cov}\left(X_{i}, X_{j}\right)=\min (i, j)$. Hence,

$$
\rho_{c}(\boldsymbol{X})=\frac{\sum_{i=1}^{d} \sum_{j<i} \min (i, j)}{\sum_{i=1}^{d} \sum_{j<i} \sqrt{i j}}=\frac{\sum_{i=1}^{d} i^{2}-\sum_{i=1}^{d} i}{\left(\sum_{i=1}^{d} \sqrt{i}\right)^{2}-\sum_{i=1}^{d} i}
$$


When we compute this formula for some values of $d$, we observe that the results completely coincide with the numerical results in Koch and De Schepper (2011). Figure 1 also shows that $\rho_{c}$ increases with $d$.

Next we study the dependence in a discounted discrete annuity as discussed in Dhaene et al. (2002b).

Example 4.2 Consider a series of deterministic payments $\alpha_{1}, \alpha_{2}, \ldots, \alpha_{d}$ due at times $1,2, \ldots, d$ respectively. At time 0 we start with a provision $V$ in order to be able to meet these future obligations. Suppose that we invest this provision and that it generates a random $(\log )$ return $Y_{i}$ in the period $[0, i]$. The provision we need at time 0 is then given by

$$
V=\sum_{i=1}^{d} \alpha_{i} e^{-Y_{i}}=\sum_{i=1}^{d} X_{i}
$$

with $X_{i}=\alpha_{i} e^{-Y_{i}}$. As in the previous example, we might assume that $\boldsymbol{Y}$ follows a multivariate normal distribution with parameters $\mathrm{E}\left(Y_{i}\right)=\mu_{i}, \operatorname{Var}\left(Y_{i}\right)=\sigma_{i}^{2}$ and $\rho\left(Y_{i}, Y_{j}\right)=\rho_{i j}$. We then find

$$
\rho_{c}(\boldsymbol{X})=\frac{\sum_{i=1}^{d} \sum_{j<i} \alpha_{i} \alpha_{j} e^{-\mu_{i}-\mu_{j}+\frac{\sigma_{i}^{2}}{2}+\frac{\sigma_{j}^{2}}{2}}\left(e^{\rho_{i j} \sigma_{i} \sigma_{j}}-1\right)}{\sum_{i=1}^{d} \sum_{j<i} \alpha_{i} \alpha_{j} e^{-\mu_{i}-\mu_{j}+\frac{\sigma_{i}^{2}}{2}+\frac{\sigma_{j}^{2}}{2}}\left(e^{\operatorname{sgn}\left(\alpha_{i} \alpha_{j}\right) \sigma_{i} \sigma_{j}}-1\right)}
$$

As a special case we consider the classic Black-Scholes model with $Y_{i}=\sum_{k=1}^{i} Z_{k}$ where the $Z_{k}$ are independent $N\left(\mu, \sigma^{2}\right)$ increments. The random vector $\boldsymbol{Y}$ then has a multivariate normal distribution with parameters $\mathrm{E}\left(Y_{i}\right)=\mu i, \operatorname{Var}\left(Y_{i}\right)=\sigma^{2} i$ and $\operatorname{Cov}\left(Y_{i}, Y_{j}\right)=\sigma^{2} \min (i, j)$. Hence, equation (11) becomes

$$
\rho_{c}(\boldsymbol{X})=\frac{\sum_{i=1}^{d} \sum_{j<i} \alpha_{i} \alpha_{j} e^{\left(-\mu+\frac{\sigma^{2}}{2}\right)(i+j)}\left(e^{\sigma^{2} \min (i, j)}-1\right)}{\sum_{i=1}^{d} \sum_{j<i} \alpha_{i} \alpha_{j} e^{\left(-\mu+\frac{\sigma^{2}}{2}\right)(i+j)}\left(e^{\operatorname{sgn}\left(\alpha_{i} \alpha_{j}\right) \sigma^{2} i j}-1\right)}
$$

Using $\rho_{c}(\boldsymbol{X})$ we can assess the effect of the parameters on the dependence in this model. In figure 2 we set $\mu=0.05, \sigma=0.2, \alpha_{i}=1(i=1, \ldots, d)$ and $2 \leq d \leq 50$. The graph shows that $\rho_{c}$ first increases with increasing dimension, but then slightly decreases from $d=8$ on. Hence, we can expect the comonotonic approximation to be less accurate for higher dimensions. Indeed, increasing the dimension in this case implies a longer time horizon and thus a weaker dependence between the ends of the discounting process. Analogous to proposition 2.13, we can conclude that the relative amount of dependence then decreases.

In figures 3 and 4 we fix $d=15$ and change the drift $\mu$ and the volatility $\sigma$ respectively. Using realistic parameter ranges, it appears that $\rho_{c}$ is still rather high but it decreases with increasing drift $\mu$. Increasing the volatility $\sigma$ has a similar effect.

\section{Aknowledgements}

Jan Dhaene, Daniël Linders and Wim Schoutens acknowledge the financial support of the Onderzoeksfonds K.U. Leuven (GOA/12/002/TBA: Management of Financial and Actuarial Risks: Modeling, Regulation, Incentives and Market Effects). Further, the authors would like to thank the referees for their valuable comments. 


\section{References}

J. Behboodian, A. Dolati, and M. Úbeda Flores. A multivariate version of Gini's rank association coefficient. Statistical Papers, 48:295-304, 2007.

M. Denuit, J. Dhaene, M. Goovaerts, and Kaas R. Actuarial Theory for Dependent Risks: Measures, Orders and Models. John Wiley \& Sons, Ltd, 2006.

J. Dhaene, M. Denuit, M. Goovaerts, R. Kaas, and D. Vyncke. The concept of comonotonicity in actuarial science and finance: Theory. Insurance: Mathematics and Economics, 31(1):3-33, 2002a.

J. Dhaene, M. Denuit, M. Goovaerts, R. Kaas, and D. Vyncke. The concept of comonotonicity in actuarial science and finance: Applications. Insurance: Mathematics and Economics, 31(2):133-161, 2002b.

J. Dhaene, D. Linders, W. Schoutens, and D. Vyncke. The herd behavior index: a new measure for the implied degree of co-movement in stock markets. Insurance: Mathematics and Economics, 50(3): 357-370, 2012.

P. Embrechts, A. McNeil, and D. Straumann. Correlation and dependence in risk management: properties and pitfalls, pages 176-223. Risk Management: Value at Risk and Beyond. M.A.H. Dempster, Cambridge University Press, Cambridge, 2002.

B. Fernández Fernández and J. González-Barrios. Multidimensional dependency measures. Journal of Multivariate Analysis, 89:351-370, 2004.

W. Hoeffding. Masstabinvariante Korrelationstheorie. Schriften des mathematischen Instituts und des Instituts für angewandte Mathematik der Universität Berlin, 5:179-233, 1940.

K. Joag-Dev. Independence via uncorrelatedness under certain dependence structures. Annals of Probability, 11(4):1037-1041, 1983.

I. Koch and A. De Schepper. Measuring comonotonicity in m-dimensional vectors. ASTIN Bulletin, 41(1):191-213, 2011.

E. Lehmann. Some concepts of dependence. Annals of Mathematical Statistics, 37(5):1137-1153, 1966.

C. Luan. Insurance premium calculations with anticipated utility theory. ASTIN Bulletin, 31(1):22-35, 2001.

A. Müller and M. Scarsini. Some remarks on the supermodular order. Journal of Multivariate Analysis, 73:107-119, 2000.

H. Panjer. Recursive evaluation of a family of compound distributions. ASTIN Bulletin, 12:22-26, 1981.

F. Schmid and R. Schmidt. Multivariate extensions of Spearman's rho and related statistics. Statistics \& Probability Letters, 77:407-416, 2007.

W. Shih and W. Huang. Evaluating correlation with proper bounds. Biometrics, 48:1207-1213, 1992.

M. Taylor. Multivariate measures of concordance. Annals of the Institute of Statistical Mathematics, 59(4):789-806, 2007.

D. Vyncke. Comonotonicity, volume 1 of Encyclopedia of Actuarial Science, pages 302-305. John Wiley \& Sons Ltd., 2004.

E.F. Wolff. N-dimensional measures of dependence. Stochastica, 4(3):175-188, 1980. 


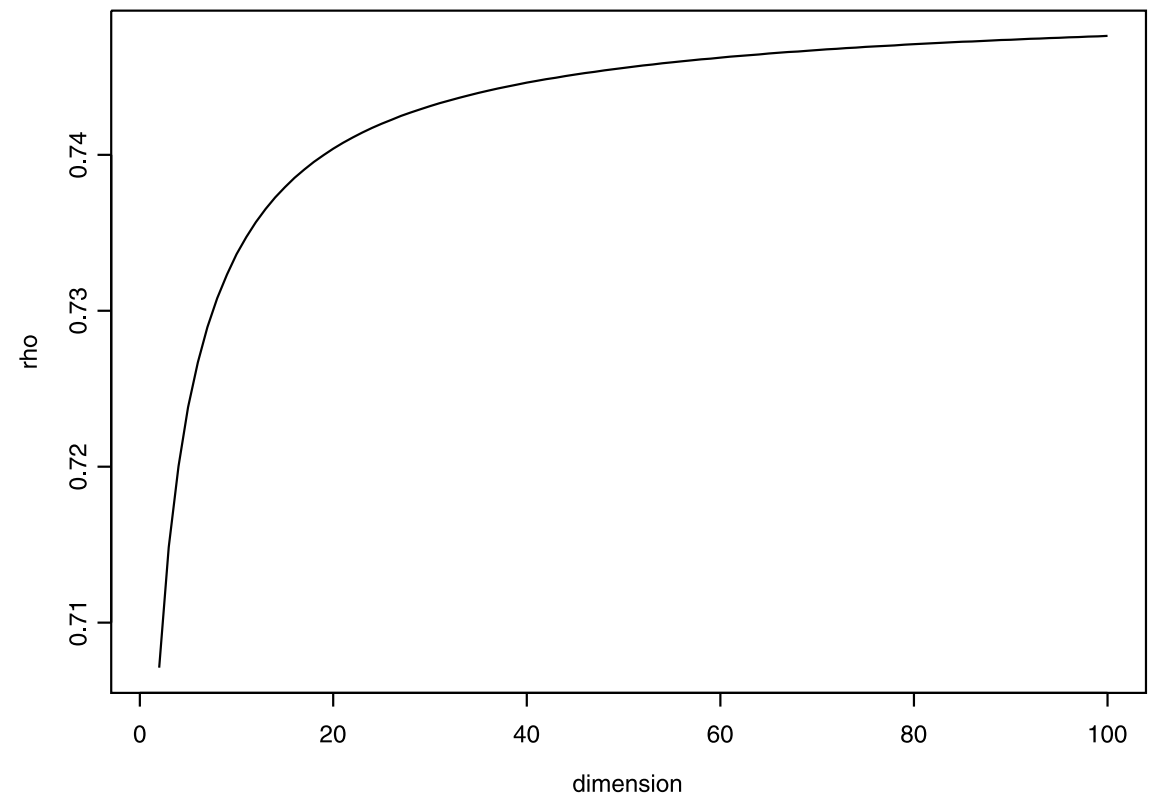

Figure 1: Multivariate normal distribution with independent increments (effect of dimension)

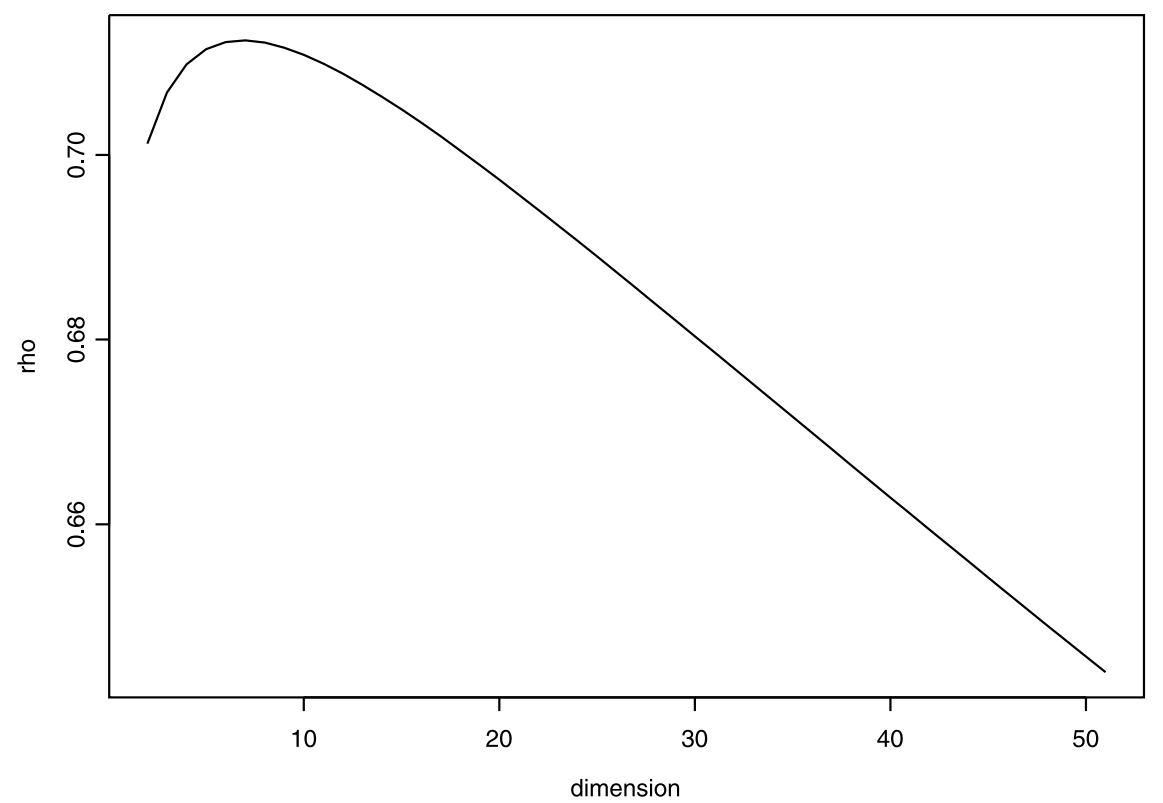

Figure 2: Lognormal discounting model for cash flows (effect of dimension) 


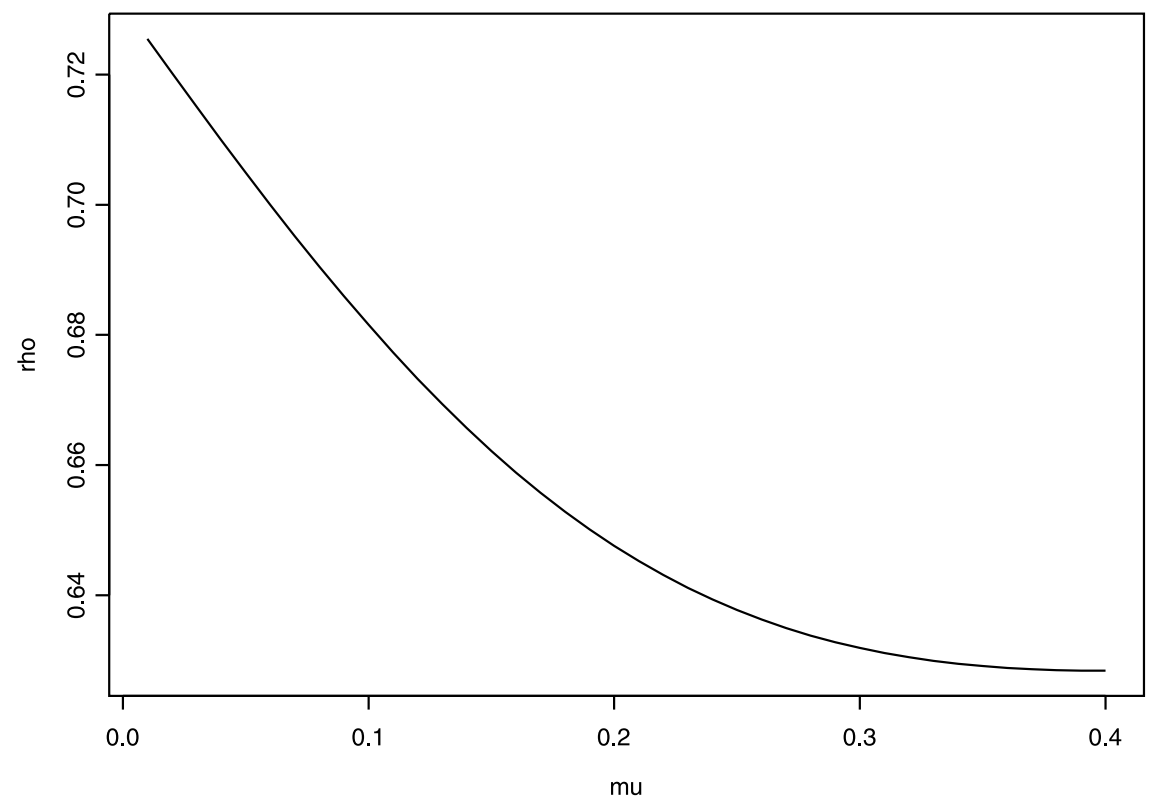

Figure 3: Lognormal discounting model for cash flows (effect of drift)

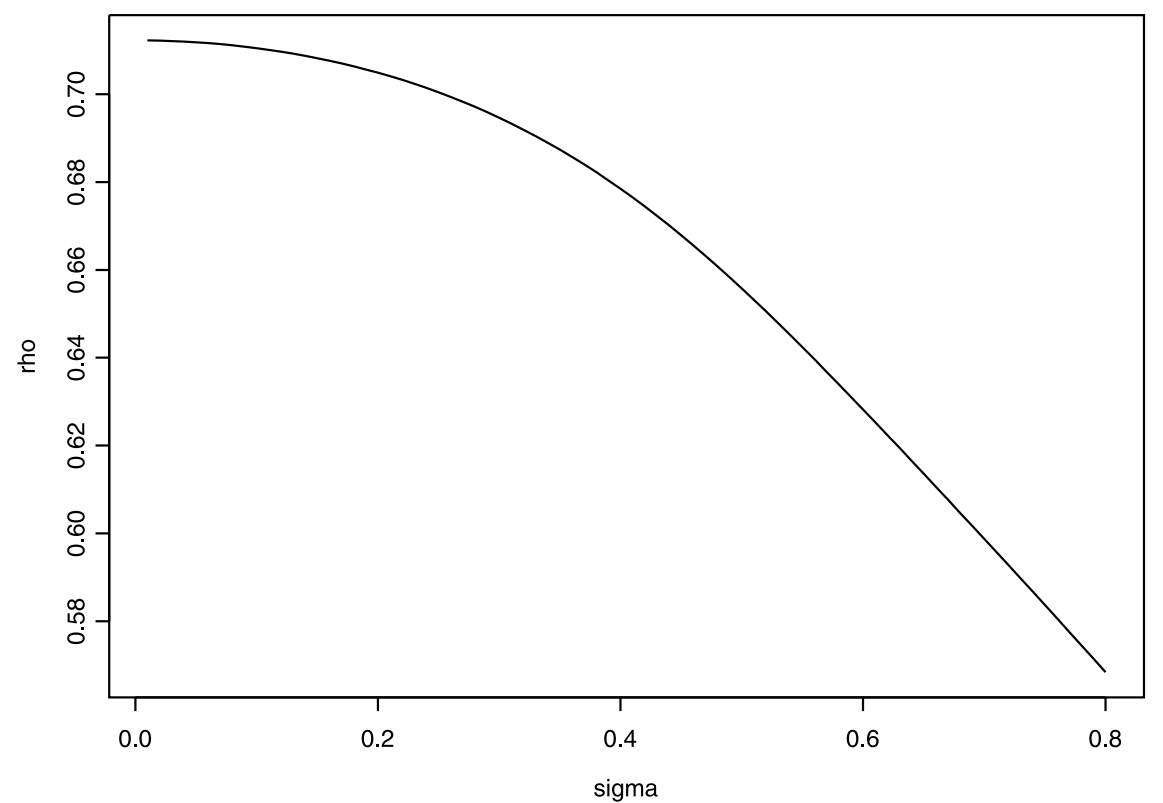

Figure 4: Lognormal discounting model for cash flows (effect of volatility) 